\title{
Cells tile a flat plane by controlling geometries during morphogenesis of Pyropia thalli
}

\author{
Kai Xu ${ }^{1,2}$, Yan Xu ${ }^{1,2}$, Dehua Ji ${ }^{1,2}$, Ting Chen ${ }^{1,2}$, Changsheng Chen ${ }^{1,2}$, Chaotian Xie ${ }^{\text {Corresp. } 1,2}$ \\ ${ }^{1}$ Fisheries College, Jimei University, Xiamen, Fujian, China \\ ${ }^{2}$ Key Laboratory of Healthy Mariculture for the East China Sea, Ministry of Agriculture, Xiamen, Fujian, China \\ Corresponding Author: Chaotian Xie \\ Email address: ctxie@jmu.edu.cn
}

Background.Pyropia haitanensis thalli, which are made of a single layer of polygonal cells, are a perfect model for studying the morphogenesis of multi-celled organisms because their cell proliferation process is an excellent example of the manner in which cells control their geometry to create a two-dimensional plane.

Methods. Cellular geometries of thalli at different stages of growth revealed by light microscope analysis.

Results. This study showed the cell division transect the middle of the selected paired-sides to divide the cell into two equal portions, thus resulting in cell sides $\geq 4$ and keeping the average number of cell sides at approximately 6 even as the thallus continued to grow, such that more than $90 \%$ of the cells in thalli longer than $0.08 \mathrm{~cm}$ had 5-7 sides. However, cell division could not fully explain the distributions of intracellular angles. Results showed that cell-division-associated fast reorientation of cell sides and cell divisions together caused $60 \%$ of the inner angles of cells from longer thalli to range from $100-140^{\circ}$. These results indicate that cells prefer to form regular polygons.

Conclusions. This study suggests that appropriate cell-packing geometries maintained by cell division and reorientation of cell walls can keep the cells bordering each other closely, without gaps. 
1 Cells tile a flat plane by controlling geometries during morphogenesis of Pyropia thalli

2

$3 \mathrm{Kai} \mathrm{Xu}^{1,2}$, Yan Xu ${ }^{1,2}$, Dehua Ji ${ }^{1,2}$, Ting Chen ${ }^{1,2}$, Changsheng Chen ${ }^{1,2}$, Chaotian Xie ${ }^{1,2} *$

$4 \quad{ }^{1}$ Fisheries College, Jimei University, Xiamen, 361021, China

$5{ }^{2}$ Key Laboratory of Healthy Mariculture for the East China Sea, Ministry of Agriculture, Xiamen,

6 361021, China

7

8 *Corresponding Author: Chaotian Xie ctxie@jmu.edu.cn 


\section{Abstract}

10 Background. Pyropia haitanensis thalli, which are made of a single layer of polygonal cells, are a perfect model for studying the morphogenesis of multi-celled organisms because their cell

12 proliferation process is an excellent example of the manner in which cells control their geometry 13 to create a two-dimensional plane.

14 Methods. Cellular geometries of thalli at different stages of growth revealed by light microscope 15 analysis.

16 Results. This study showed the cell division transect the middle of the selected paired-sides to 17 divide the cell into two equal portions, thus resulting in cell sides $\geq 4$ and keeping the average number of cell sides at approximately 6 even as the thallus continued to grow, such that more than $90 \%$ of the cells in thalli longer than $0.08 \mathrm{~cm}$ had 5-7 sides. However, cell division could not fully explain the distributions of intracellular angles. Results showed that cell-division-associated fast reorientation of cell sides and cell divisions together caused $60 \%$ of the inner angles of cells from longer thalli to range from $100-140^{\circ}$. These results indicate that cells prefer to form regular 23 polygons.

Conclusions. This study suggests that appropriate cell-packing geometries maintained by cell division and reorientation of cell walls can keep the cells bordering each other closely, without gaps. 


\section{Introduction}

Pyropia is an intertidal red algae. Its edible portion forms during the thallus stage and has an annual production worth about 1.3 billion USD (Blouin et al. 2011). The thallus is a membranous sheet in a lanceolate shape made of one or two layers of cells. Two of the most economically important cultured species, Pyropia yezoensis and P. haitanensis, only contain one layer of cells. During the thallus stage, $P$. haitanensis thalli can take on three morphologies in sequence: singlecelled conchospores (point), linearly ordered groups of 4 to 10 cells (line), membranous sheet (plane). The cell proliferation during morphogenesis of $P$. haitanensis thalli is essentially twodimensional (2D) expansion on a plane. The specific geometries make P. haitanensis a simple but valuable model organism for the study of the morphogenesis of multi-celled organisms. Although the cell-packing geometries keep changing due to cell growth and division, most of the cells could be considered convex polygons with a small number of spherical cells at the base. The morphogenesis of thalli features cells that border each other closely with no empty spaces or gaps. The mechanisms underlying this feature are equivalent to a mathematical question regarding how convex polygons tile or tessellate in regular patterns on 2D planes. The geometric patterns of Pyropia cells also follow the mathematical laws and must be tightly controlled, but the patterns and underlying control mechanisms are poorly understood.

Three laws were here generalized for the analysis of general topological properties of 2D tessellation: Euler's law (faces - edges + vertex =1), Lewis' law (the relationship between mean area of a convex n-sided cell and n) and Aboav-Weaire law (Aboav 1980) (the relationship between the mean number of sides of neighboring cells of a convex n-sided cell and n) (Aboav 1980; Lewis 1928; Sanchez-Gutierrez et al. 2016; Weaire \& Rivier 1984). Two basic mathematical generalizations were found to underlie the tessellations in which only one kind of polygon was used to tile a flat plane (Grünbaum \& Shephard 1987; Lord 2016): 1. Any kind of polygon with more than 6 sides would be unable to form a close tile pattern on a flat plane. 2. To date, 15 irregular pentagons, 16 hexagons (including regular hexagon) and all triangles and quadrilaterals have been confirmed to be able to form close tile patterns on flat planes. However, the tessellation of Pyropia thalli is the tiling of a flat plane using more than one kind of polygon due to growth and cell division changing the cell-packing geometries. Conserved distribution of cellular polygons has been observed in many proliferating tissues. It generally features a predominance of hexagonal cells and an average of 6 sides, and it is considered as a mathematically determined 
consequence of cell proliferation (Gibson et al. 2006; Graustein 1931; Lewis 1926; Lewis 1928). However, a recent study reported that many different natural tissues have very different distributions of polygons (Sánchez-Gutiérrez et al. 2016).

During the past few decades, few studies have focused on cell-packing geometry, mostly by studying the epithelial cells of Drosophila wings, which can transition from irregular arrangements to hexagonal patterns before hair formation (Classen et al. 2005; Farhadifar et al. 2007; Gibson et al. 2006). It is still under debate whether the cellular geometry is achieved by cell division (Gibson et al. 2006) or cell rearrangement mediated by the physical properties of the cells (Classen et al. 2005; Farhadifar et al. 2007). A recent work by Sánchez-Gutiérrez et al. (2016) has shed light on the mechanism that drives packed tissue organization. Their paper demonstrated an interesting finding, that sections of muscle fiber (a natural non-proliferative tissue) can be enriched in hexagons. They found that a physical constraint that induced by the balance of forces between cells in non-proliferative and proliferative tissues can cause all packed tissues to be arranged in a manner similar to Voronoi diagrams. Thus, Sánchez-Gutiérrez et al. (2016) suggested that conserved polygon distribution does not necessarily need the proliferation mechanisms.

In a model study, Patel et al. (2009) found that strong division symmetry is required to reproduce the predominance of hexagonal cells, which indicates that the orientation of the mitotic cleavage plane was not random. The surface energy and tension of cell membrane were mainly determined by the shape of protoplasm; these biophysical properties were found to change the physiological performance and gene expression (Chen 2008; Ingber et al. 2014). In this way, the interior angles of the polygonal cells not only determined the bordering patterns of cells but could also change the force balance between cells. Thus, if the initial cellular geometries of two daughter cells were determined solely by the original geometries of mother cell and the orientation of the cleavage plane, then the force balance would be disturbed by formation of the interior angles of daughter cells. In this study, to understand the dynamic processes and mechanisms underlying cellpacking geometries during morphogenesis of $P$. haitanensis thalli, samples collected during different stages of growth were used for analysis.

\section{Materials and methods}

The materials used in this study were 3 strains of $P$. haitanensis present: Z-61 (red-brown), G-2 (green), and O-9 (orange). These strains were selected by the Laboratory of Germplasm 
Improvements and Applications of Pyropia in Jimei University, Fujian Province, China. Ripened free-living conchocelis of all 3 strains were cultured in natural seawater at $21^{\circ} \mathrm{C}$ and $50 \mu \mathrm{mol}$ photons $\mathrm{m}^{-2} \mathrm{~s}^{-1}(12: 12, \mathrm{~L} / \mathrm{D}$ cycle). The culture media were natural seawater enriched with nutrients according to Provasoli's enrichment solution (PES) and refreshed every 2 days. The culture media bubbled continuously using filter-sterilized air to promote the liberation of conchospores. Thalli arose from meiosis after conchospore germination. Healthy thalli were investigated and photographed under a microscope (Nikon U20).

Cells located at the base of the thallus were excluded from the following analysis because they were too circular in form. Only cells completely surrounded by other cells were selected. Because the thalli were considered a 2D plane in this study and all the convex polygon shaped cells of the thalli bordered each other closely, then the geometries of the polygonal cells were quantified to analyze the tessellation of the 2D thalli. The cell sides were counted, and the measurements of cell area (polygon area), side length, and intracellular angles (interior angles of the polygonal cell) were made using AmScope Toupview 3.0. If the length of a boundary exceeded $1 \mu \mathrm{m}$ (cell wall $\approx 0.5-1 \mu \mathrm{m})$, then that boundary was considered a side. To help count the sides of the cells, photos were treated with 2 color effects (black and white and/or inversion) using Google Picasa 3.0 software.

Cell division was identified using time-series observation. The daughter cells were assumed to have the same height, and the size ratio of daughter cells was calculated as $\mathrm{SR}=\mathrm{As} \div \mathrm{Ab}$, where As and $\mathrm{Ab}$ are area of the smaller and larger daughter cells, respectively. Cell division section was calculated as DS $=|\mathrm{S} 1 \div(\mathrm{S} 1+\mathrm{S} 2)-0.5|$, where S1 and S2 are the lengths of daughter sides (not the shared side) of two daughter cells. The value of the division section ranged from 0-0.2, meaning that the sides were divided through the middle. To quantify the relative position of the paired sides that were transected by cell divisions on the polygonal cells, two sets of interval sides between the paired sides were counted from the opposite directions at the point in time before division, and the smaller number was used in this study. More than 10 cells and divisions were examined for each thallus, and the results were expressed as mean $\pm \mathrm{SD}$.

\section{Results}

Conchospores are single cells that attach to the surfaces of glass culture bottles immediately after liberation. Conchospores first germinate into young thalli from meiosis after the formation of 
121 polarization (Fig. 1A). These young thalli contain only linearly ordered cells which were produced 122 after sequential divisions in transverse orientation (Fig. 1B), and then expand on a two-

123

124

125

126

127

128

129

130

131

132

133

134

135

136

137

138

139

140

141

142

143

144

145

146

147

148

149

150

151 dimensional plane to form a membranous sheet made of a single layer of cells. During early development, none of the cells was fully bordered by others (Fig. 1A-D). As the thallus grows, more and more cells fully bordered by others (Fig. 1). Although thalli retain a rough lanceolate morphology, the cells at opposite positions on the same thallus can show different geometric patterns, different numbers of cells, or both (Fig. 1D, F). The entire developmental stages of Pyropia haitanensis thalli are shown in a diagram (Fig. 1G).

The number of sides of $P$. haitanensis thalli cells ranged from 4 to 9 ( $n=6,434$ cells, Fig. 2A, B). Typically, cells with 5 sides were the most common during the initial stage of growth, but they become less as the thallus increases in length, supplanted by 6-sided cells (Fig. 2A). Regardless of the color of the thallus, 6-sided cells were the most prevalent in thalli longer than $0.08 \mathrm{~cm}$, and $90 \%$ of the cells had 5 to 7 sides (Fig. 2B; Fig. S1). The distribution of angles was also closely related to thallus length. Cellular inner angles were in range of $45-176^{\circ}(n=2,521$, Fig. $2 \mathrm{C}, \mathrm{D})$. The peaks of angle distributions shift from range of $90-110^{\circ}$ to $110-130^{\circ}$ along with the elongation of thallus, which was consistent with changes on average cell sides (Fig. 2C, D). The average numbers of cell sides in thalli shorter than $0.08 \mathrm{~cm}$ ranged in 5.0-5.69 and that of longer thalli were 5.72-5.97 (Fig. 2E). The average numbers of cell sides in thalli increased to almost 6 along with the growth of thallus. The average intracellular angles of thalli, which were longer than 0.08 $\mathrm{cm}$, ranged in $112-119^{\circ}$; the average angles of shorter thalli were in range of $103-110^{\circ}$ (Fig. $2 \mathrm{~F}$ ). Thus, when the average numbers of cell sides were increased to almost 6 , the average angles approached to approximately $120^{\circ}$, which is the average angle of hexagons. In addition, angles within the range of $100-140^{\circ}$ accounted for $>60 \%$ in thalli longer than $0.08 \mathrm{~cm}$ (Fig. 2D). The distributions and average values of these geometries suggested that cells favored regular polygons.

Cell divisions were studied through time-series observation. Most of the cells $(93 \pm 6 \%$, a total of 187 cells in 8 thalli were examined) that before division were 5- to 7-sided cells (Fig. 3A). In the polygonal cells, cell divisions preferred to transect unconnected paired-sides (99\%), and 97\% of the paired-sides were intervened by 1 or 2 sides before division (Fig. 3B). In addition, most of the division sections $(79 \%)$ were $<0.2$ and the average division section was $0.13 \pm 0.03$ (267 cells in 8 thalli were examined, Fig. 3C). These observations suggested that mitosis preferentially produced two daughter cells of the same size, this speculation was confirmed by the results of the 
152 current work that the size ratios of the smaller daughter cells to the larger ranged mainly $(90 \%)$,

153 between 0.7 and 1 and the average size ratio was $0.86 \pm 0.03$ (227 cells in 9 thalli were examined, 154 Fig. 3D).

155 Cell division always produced a new border shared by two daughter cells (Fig. 4A-D), and 156 they preferentially produced 3-fold junctions, which accounted for $99 \pm 1 \%$ of the total (782 157 junctions in 5 thalli were examined, Fig. 4E). Because the cells of $P$. haitanensis thalli are packed 158 by rigid cell walls, the cell neighbor relationships remained stable until the topology was disturbed 159 by the formation of two daughter cells by cell division (Fig. 4A-D). Cell division was found to 160 new angles formed in neighbor cells at a rate of $20 \pm 3^{\circ}$ per day over 2 days after the start of cell division (138 angles in 8 thalli were examined, Fig. 4A-D, F).

\section{Discussion}

Lewis (1926) found that the average number of sides per polygonal cell to be close to 6 in cucumber skin. The core mechanism of this kind of phenomena was established by Graustein (1931): The number of sides that meet at every vertex is always 3 . Then the tessellation with convex polygonal cells followed Euler's law to achieve an average of 6 sides. In this way, cell division must prefer to produce 3-fold junctions (vertexes) rather than junctions common to 4 or more polygonal cells. Gibson et al. (2006) also reported that the average number of sides of Drosophila wing cells remained close to 6, consistent with Euler's law, and they successfully used a Markov chain model validated with characteristics of cell division to predict the overall distribution of polygonal cell types. For this reason, they proposed that mechanisms of cell division could be mathematically sufficient to explain the predominance of 6-sided cells and the average 6 sides.

Results also showed the average number of sides of $P$. haitanensis cells increased to almost 6 as the thallus continued to grow, and the distribution of the number of sides in longer thalli remained very close to the measured and predicted values of Drosophila wings (Fig. 2B) (Gibson et al. 2006). Thus, the assumed conditions of Markov chain model are the major cause of mathematically achieved hexagonal cell patterns in multicellular organisms (Gibson et al. 2006). Based on previous studies and on the current results, the assumed conditions were reorganized as follows (Gibson et al. 2006; Graustein 1931; Lewis 1926; Lewis 1928): 1. Cells bordered each 
183

184

185

186

187

188

189

190

191

192

193

194

195

196

197

198

199

200

201

202

203

204

205

206

207

208

209

210

211

212

213

other closely and grew uniformly as they proliferated on the flat plane. 2. Cell neighbor relationships were stable. 3. Cell division preferentially formed tricellular junctions. 4. Cell division favored equal division of cells by forming a new side shared by the two daughter cells.

Conditions 1-2 were supported by previous studies and the current observations (Gibson et al. 2006; Graustein 1931; Lewis 1926; Lewis 1928). Cell rearrangement has been proposed as a primary mechanism to control the formation of cell hexagonal packing in Drosophila wings, which resulted in exchanges of neighbor cells (Classen et al. 2005; Farhadifar et al. 2007; Heller et al. 2016). Gibson et al. (2006) did not detect cell rearrangement in proliferating Drosophila wing cells. However, a recent study including automated, systematic high-throughput analysis methods showed 13 times of cell rearrangement per 1,000 cells per hour (Heller et al. 2016). The cells of $P$. haitanensis thallus are packed by rigid cell walls the same way as the plant cells, which indicates that each walled cell and its neighbor cells adhere to each other to prevent cell migration (Taiz \& Zeiger 2010). The assumed condition 2 is suitable to the current study on the thalli of the macroalgae $P$. haitanensis and previous studies on the higher plant cucumber (Graustein 1931; Lewis 1926; Lewis 1928).

Because of the stable cell neighbor relationships (e.g. Fig. 4), the formation of tricellular junctions was found to depend on the cut position of the divide during cell division: 1 . Cell division always cut through two sides of a cell rather than two vertexes or one vertex and one side. In this study, only $<1 \%$ of observed cells were divided through both a vertex and a side (Fig. 4C), and none were cut through two vertexes of a polygonal cell. 2. Cell division favored division at different positions to produce tricellular junctions. That is supported by our observations that only 3-and 4-fold junctions were identified and tricellular junctions accounting for $99 \%$ (Fig. 4E).

Under conditions 1-3, the paired sides of mother cells were found to split into 4 sides which it shared with neighboring cells, while a new side would form, shared by two daughter cells (Fig. 4A-D). This increased the number of cells by one due to the division, but the net increase of shared sides was 3. In this way, every division increased the number of sides by 6 . The average number of cell sides would then approached 6 because of the large number of cell divisions taking place during the growth of the thallus (Fig. 2E). For the same reason, the average number of sides per cell would be closes to 3 if cell division cut through vertexes or closes to 4 if cell divisions favored producing 4-fold junctions. As for the non-walled cells of 
214 epithelia, the assumptions used in Markov chain model (Gibson et al. 2006) are challenged by

215 the observations of cell rearrangements (Classen et al. 2005; Heller et al. 2016). However,

216 whatever cell rearrangements may be ignored or emphasized, the overall distribution of

217 polygonal cell in epithelial tissues (Classen et al. 2005; Gibson et al. 2006; Heller et al. 2016) is

218 very close to walled cells in the present study and previous studies (Graustein 1931; Lewis 1926;

219 Lewis 1928). Because the cell rearrangements resulted in cells with a larger number of sides that

220 lose an edge to cells with a smaller number of sides (Heller et al. 2016), it is possible that cell

221 rearrangements would not change the distribution of polygonal cells of the epithelial tissue on a

222 global scale, but further tests are needed to confirm this hypothesis.

However, conditions 1-3 were not found to necessarily determine the distributions of polygonal cells. The Markov chain model was found to predict the distributions of 5- to 7-sided cells very well by leaving out 3 - and 4 -sided cells due to their low prevalence $(\approx 0$ and $\approx 4 \%$, respectively) upon real observation (Gibson et al. 2006). If the orientation of cleavage was random, then the rate at which 2 connected sides ( 0 interval side) would transect by cell division would be roughly $60 \%, 43 \%, 35 \%$, and 30\% for 4-, 5-, 6-, and 7-sided cells, respectively. However, in both Drosophila wing cells (Classen et al. 2005; Farhadifar et al. 2007; Gibson et al. 2006), cucumber skin cells (Lewis 1926; Lewis 1928), and longer P. haitanensis thalli, the prevalence of 4-sided polygonal cells was always $<5 \%$. Even though cells would gain an average of one side each time from its neighbors divided (Gibson et al. 2006), a larger proportion of 4-sided cells would still be expected to be found under real conditions. In this way, the measured values contradict the assumption that cell division would be randomly orientated (Gibson et al. 2006).

Biological processes and mechanisms are also more complex than had once been thought. It seems to make sense that cells would divide equally during mitosis. Theoretically, if cell division prefers to divide 5-7-sided regular polygons in half, then the cleavage plane should always cut unconnected paired sides. The present study confirmed this speculation (Fig. 3B). The current study is consistent with a previous model study by Patel et al. (2009). They found different cleavage patterns generated different distributions of polygonal cells, and their results suggested that strong divisional symmetry is required to reproduce the predominance of hexagonal cells. In addition, cell division was found to favor cutting in the middle of the sides (Fig. 3C). In this way, to achieve the equal division, both the relative position of paired sides that were cut by cell division and the points at which they were cut were selected (Fig. 3D). This shows that the reason for cell 
245 sides $\geq 4$ was that mitosis favored equal division of cells, which could help improve the 246 assumptions of the Markov chain model (Gibson et al. 2006). Moreover, $0.6 \%$ of paired sides (187 247 cells in 8 thalli were examined) were connected and transected by cell division, which was 248 expected to take place in $0.6 \%$ of triangular cells. However, all of the examined cells $(n=6,432)$ 249 had 4 sides or more. The creation of triangular cells was prevented by gain of sides from divisions 250 of neighbor cells (Fig. 4D).

251 It must be emphasized the number 6 is just a statistical average. The number of sides of an 252 individual cell is not a fixed number, but rather fell mainly within the range of 4 to 9 (Fig. 2). The 253 increase in the average number of cell sides (Fig. 2E) reflected the kinetic geometry of all the 254 polygonal cells on a global scale during the development of P. haitanensis thalli. Each cell in the 255 tissue needed to cooperate or deal with other cells to help maintain appropriate geometry. For 256 example, in the $2 \mathrm{D}$ tessellation, the sum of angles around a multi-cellular junction must equal $360^{\circ}$. Furthermore, the mathematical laws of 2D tessellation suggested that the geometry of individual cells is not random, especially when the tessellation was characterized by specific distributions of polygonal cells and the average number of sides approached 6 as the number of cells increased (Aboav 1980; Grünbaum \& Shephard 1987; Lewis 1928; Lord 2016; Weaire \& Rivier 1984). So, if the geometry of individual cells were random or controlled only by each cell itself, then it would be quite possible that a group of cells would not tile a flat plane. However, during this study, healthy thalli of $P$. haitanensis did not contain holes or gaps between cells. In addition, if intracellular angles remain stable, then newly formed angles would be determined solely through cell division, which means that the new angles of the two daughter cells should have equal chances to be either $\leq 90^{\circ}$ or $\geq 90^{\circ}$, and angles of $180^{\circ}$ would be formed inside neighbor cells. However, the majority of inner angles were within the range of $100-140^{\circ}$ when cell sides ranged in $5.72-$ 5.97, and only minor amount of angles were observed $<60^{\circ}$ or $>170^{\circ}$ (Fig. 2B and 2D). These findings could not be explained by cell division alone.

Newly formed tricellular junctions were located exactly at the cut points on paired sides because of cell division (Fig. 4). Time series observation indicated that sides were reoriented around each newly formed junction, resulting in adjustments of angles which was associated with these sides, and the newly formed angles within neighbor cells showed a decrease rate of $20^{\circ}$ per day during cell division. In this way, cell division and reorientation of sides were the primary reasons for the distribution of intracellular angles (Fig. 2, 3). The adjustments of intracellular 
276 angles indicated that cells of $P$. haitanensis thalli cooperate with each other by favored the

277 formation of regular polygonal patterns. The cell walls in regular polygonal patterns could render

278 the shape of protoplasm more spherical, which could decrease the surface energy (Chen 2008;

279 Ingber et al. 2014). Thus, the reorientation may have been mediated or controlled by biophysical

280 properties.

281

Recently, by use of Voronoi diagrams, a surprising study by Sánchez-Gutiérrez et al. (2016)

282 proposed a new mechanism that, independent of cell proliferation mechanisms, is through physical

283 cellular constraints drives the distribution of polygonal cells. Each convex polygonal cell is a

284 Voronoi cell, generated from a specified point (called a seed). Each such cell is a region consisting

285 of all points closer to its seed than to any other seed. The current study showed that cell division

286 cut through the middle of the selected paired sides to produce equal-sized daughter cells, which

287 means our findings could offer constraints for using Voronoi diagrams to mimic the cellular

288 proliferation processes by locating the positions of seeds in daughter polygonal cells. Voronoi

289 tessellation has an obvious advantage over cell proliferation mechanisms in that the former could

290 explain the conserved polygon distribution in non-proliferative tissue (Gibson et al. 2006;

291 Graustein 1931; Patel et al. 2009; Sánchez-Gutiérrez et al. 2016; and the current study). The

292 differences and connections between the mechanisms dependent and independent of cell

293 proliferation require further study.

294

295

\section{Conclusion}

296 The evolution of multi-celled organisms was a tremendous step in the early history of life,

297

298

299

300

301

302

303

304

305

306 but the mechanisms by which the cells are arranged to form organisms with specific morphology are still unclear. $P$. haitanensis inhabits the intertidal zone which is subject to fast and periodic changes in temperature, seawater, light, and nutrient availability. Thus, this macroalgae species needs to tightly, precisely, and effectively control their growth and cell division to maintain their typical morphological features. The current study indicated that cell division mainly involved cutting through middle of selected paired sides to mathematically bring the average number of sides per cell increased to almost 6 as the thallus elongated; cellular angles changed through reorientation of cell sides, which, with cell division, determined the distribution of angles. These results offer some help in understanding that the physiological and geometric performances of a single cell exhibit a global pattern in a multicellular organism. 


\section{References:}

Aboav DA. 1980. The arrangement of cells in a net. Metallography 13:43-58.

Blouin NA, Brodie JA, Grossman AC, Xu P, and Brawley SH. 2011. Porphyra: a marine crop shaped by stress. Trends in Plant Science 16:29-37.

Chen CS. 2008. Mechanotransduction - a field pulling together? Journal of Cell Science 121:32853292. 10.1242/jcs.023507

Classen A-K, Anderson KI, Marois E, and Eaton S. 2005. Hexagonal packing of Drosophila wing epithelial cells by the planar cell polarity pathway. Developmental cell 9:805-817.

Farhadifar R, Röper J-C, Aigouy B, Eaton S, and Jülicher F. 2007. The influence of cell mechanics, cell-cell interactions, and proliferation on epithelial packing. Current Biology 17:20952104.

Gibson MC, Patel AB, Nagpal R, and Perrimon N. 2006. The emergence of geometric order in proliferating metazoan epithelia. Nature 442:1038-1041.

Grünbaum B, and Shephard GC. 1987. Tilings and patterns. New York: Freeman Press.

Graustein W. 1931. On the average number of sides of polygons of a net. Annals of Mathematics:149-153.

Heller D, Hoppe A, Restrepo S, Gatti L, Tournier AL, Tapon N, Basler K, and Mao Y. 2016. EpiTools: an open-source image analysis toolkit for quantifying epithelial growth dynamics. Developmental cell 36:103-116.

Ingber DE, Wang N, and Stamenovic D. 2014. Tensegrity, cellular biophysics, and the mechanics of living systems. Reports on Progress in Physics 77:046603. 10.1088/0034$4885 / 77 / 4 / 046603$

Lewis FT. 1926. The effect of cell division on the shape and size of hexagonal cells. The Anatomical Record 33:331-355.

Lewis FT. 1928. The correlation between cell division and the shapes and sizes of prismatic cells in the epidermis of cucumis. The anatomical record 38:341-376.

Lord N. 2016. Constructing the 15th pentagon that tiles the plane. The Mathematical Gazette. Cambridge: Cambridge University Press, 154-158.

Patel AB, Gibson WT, Gibson MC, and Nagpal R. 2009. Modeling and inferring cleavage patterns in proliferating epithelia. PLOS Computational Biology 5:e1000412. 
338

339

340

341

342

343

344

345

346

347

348

10.1371/journal.pcbi. 1000412

Resino J, Salama-Cohen P, and García-Bellido A. 2002. Determining the role of patterned cell proliferation in the shape and size of the Drosophila wing. Proceedings of the National Academy of Sciences 99:7502-7507.

Sanchez-Gutierrez D, Tozluoglu M, Barry JD, Pascual A, Mao Y, and Escudero LM. 2016. Fundamental physical cellular constraints drive self-organization of tissues. EMBO Journal 35:77-88. 10.15252/embj.201592374

Taiz L, and Zeiger E. 2010. Plant physiology. 5th. Sundeland, Massachusetts: Sinauer Associates. Weaire D, and Rivier N. 1984. Soap, cells and statistics_random patterns in two dimensions. Contemporary Physics 25:59-99. 10.1080/00107518408210979 


\section{Figure 1}

Morphogenesis of $P$. haitanensis during the thallus stage.

(A) Polarized conchospore. (B) Young thalli contained linearly ordered groups of 5 cells due to cell division alone along the apical-basal axis. The basal cells along the right side were elongated into rhizoids. (C-F) Cell proliferation of P.haitanensis thalli along a two-dimensional plane. White arrows showed different tessellation patterns at symmetrical positions on the same thallus. All scale bars are $20 \mu \mathrm{m}$. (G) All developmental stages. 

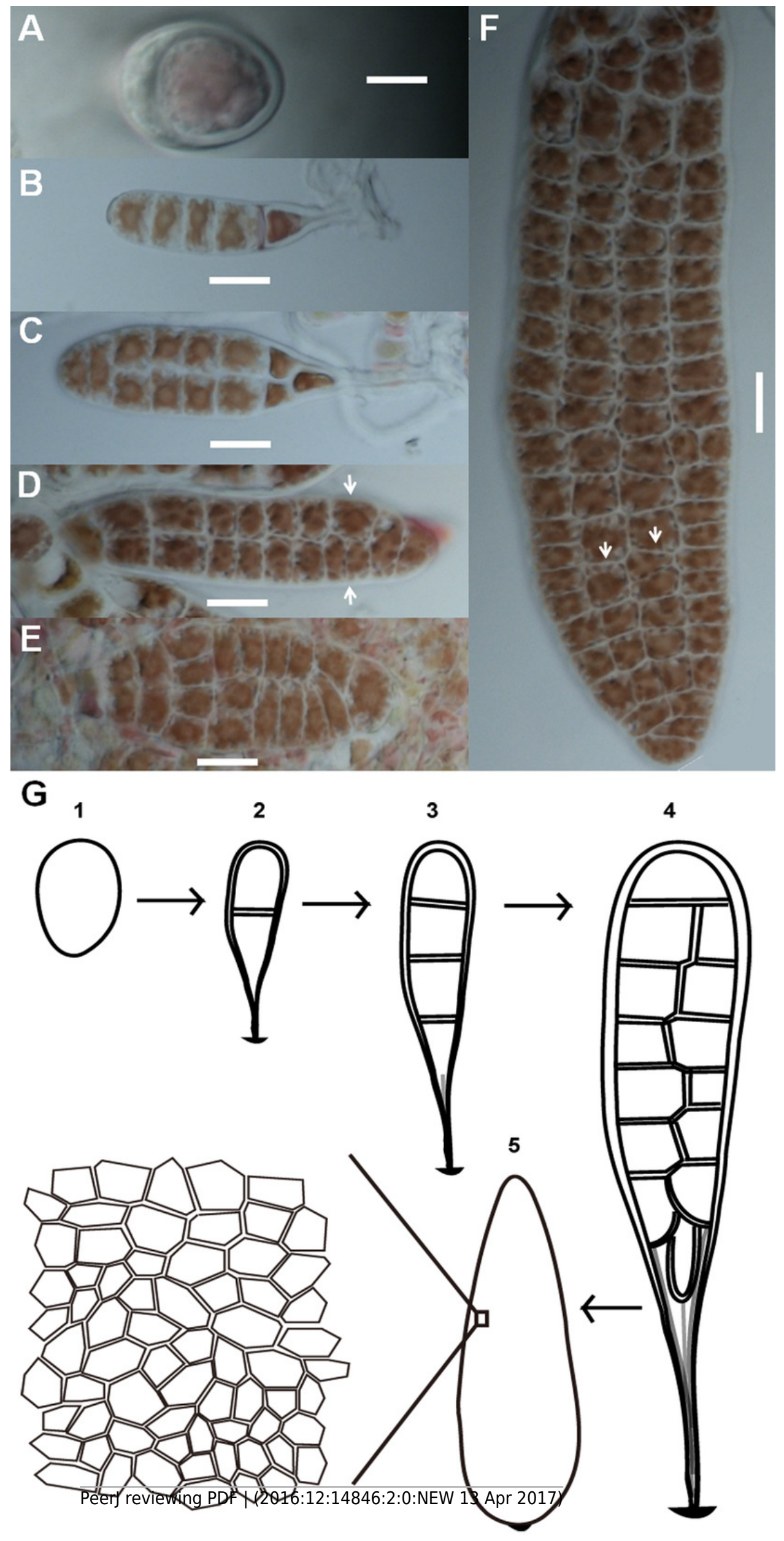
Figure 2 (on next page)

Relationship between thallus length and cell-packing geometries.

(A-D) Typical distributions of (A, B) number of cell sides and (C, D) intracellular angle of the thallus at different lengths. (E-F) Average (E) number of cell sides and (F) intracellular angle of the thallus at different lengths. Symbols in red-brown, green, and orange represent the color of 3 strains of $P$. haitanensis Z-61, G-2, and 0-9, respectively. Sample numbers are shown in parentheses. 

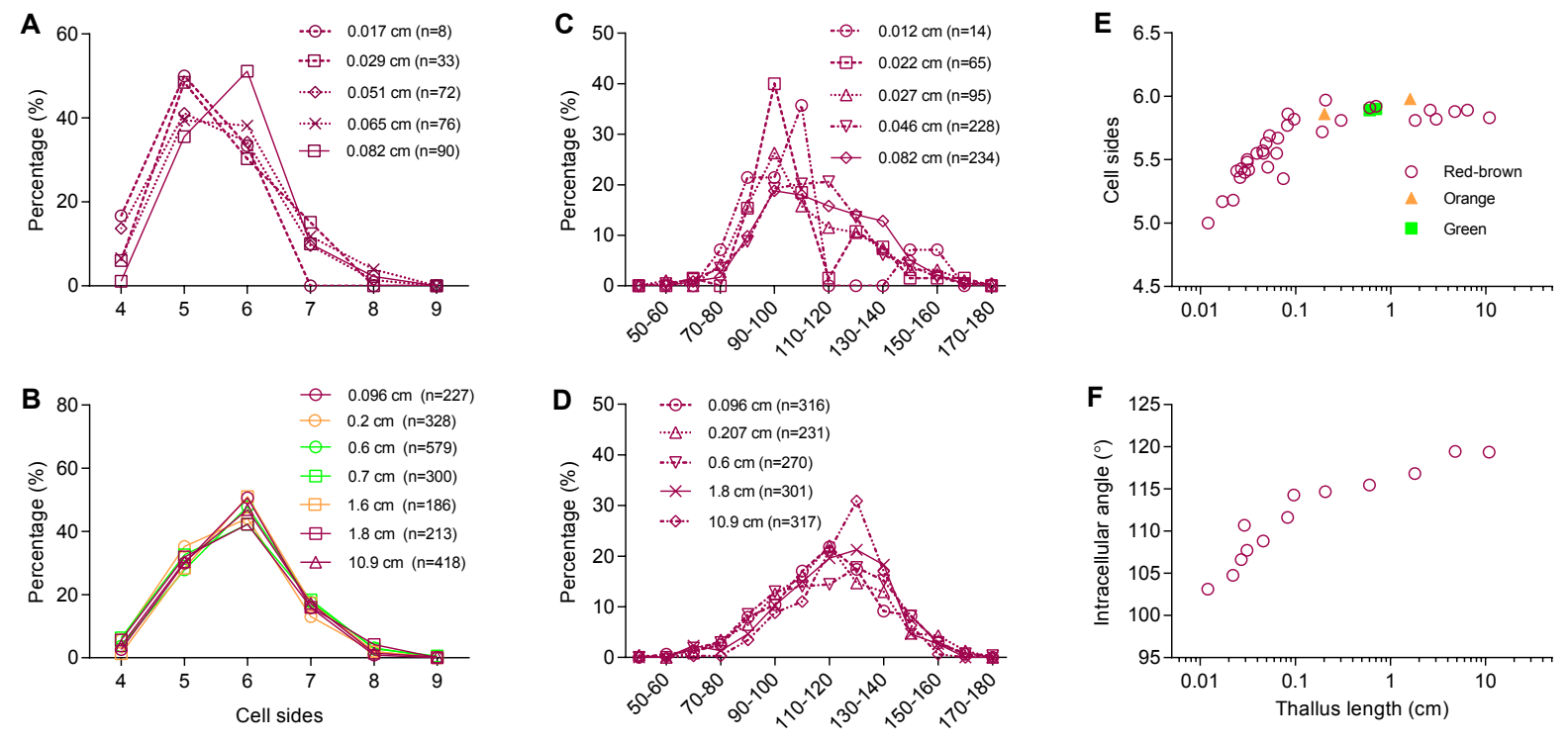

Intracellular angle $\left({ }^{\circ}\right)$ 


\section{Figure $\mathbf{3}$ (on next page)}

Cell division cut at middle of selected paired-sides.

(A) Distribution of cell sides before cell division (187 cells in 8 thalli were examined). (B) Cell division favoring transection of unconnected paired sides (99\%). The interval sides between paired sides indicates the relative positions on a polygonal cell before cell division, here the smaller of the two numbers enumerated from opposite directions. (C-D) Distributions of (C) division sections (267 cells in 9 thalli were examined) and (D) size ratios of daughter cells (227 cells in 9 thalli were examined). Division section indicates the relative distance between midpoint of a side and transection position on that side. The size ratios of daughter cells referred to the ratio of the small daughter cell to the big one. Sample numbers are shown in parentheses. The data are expressed as mean \pm SD. 

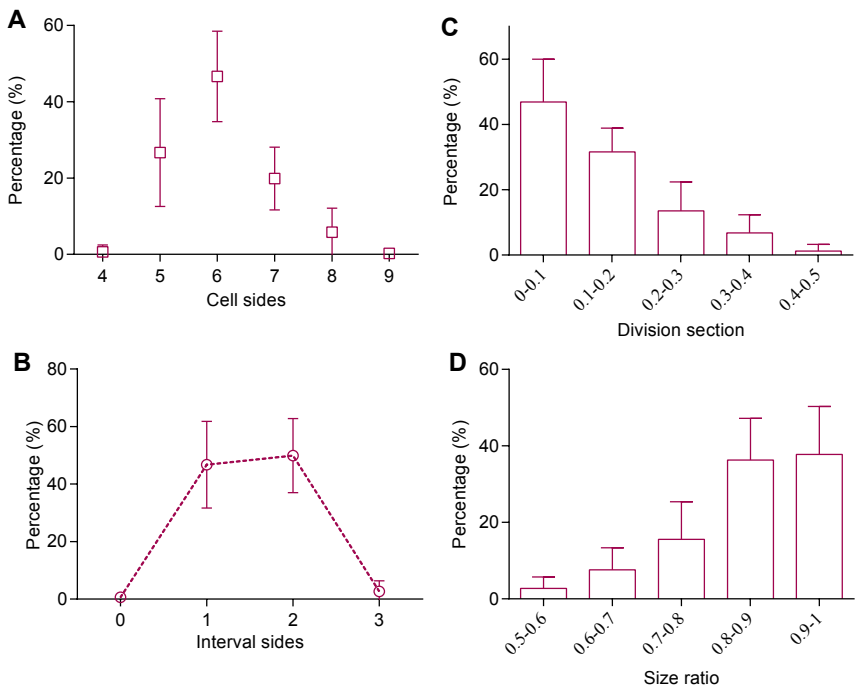


\section{Figure 4}

Cell division associated reorientation of cell walls.

(A-D) White arrows point to the newly formed junctions and angles due to cell division. Due to reorientation, the new angles that formed in neighbor cells decreased during the first $48 \mathrm{~h}$ after the beginning of cell division. In general, the reorientation remained for about 2 days until the boundary between two daughters became clear. All scale bars are 10 $\mu \mathrm{m}$. (A-B) Cell division transecting unconnected 2 sides. (C) Cell division transecting a side and a vertex of an 8-sided cell, which produced a 4-fold junction. (D) Cell division transecting connected 2 sides. Arrows indicate where the daughter cell gained two sides from dividing neighbor cells, which prevented the creation of triangular cell. (E) About $99 \pm 1 \%$ of junctions were 3 -fold and the rest were 4-fold. (F) Diagram of cell division of $P$. haitanensis thallus. A cell in blue color with $\geq 4$ sides, its paired-sides which shared with two red cells would be transected by cell division. Cell division increased the number of cells by 1 and the number of counted cell sides by 6 . The reorientation of cell walls around a newly formed junction led to adjustments of 1 angle ( $a$ ) in neighboring cells and 2 angles ( $b$ and $c$ ) in the daughter cells. During the first 2 days, the rate of decrease of the new angles in neighbor cells was $20 \pm 3^{\circ}$ per day (angle $a$, 138 angles in 8 thalli were examined). The data are expressed as mean \pm SD. 


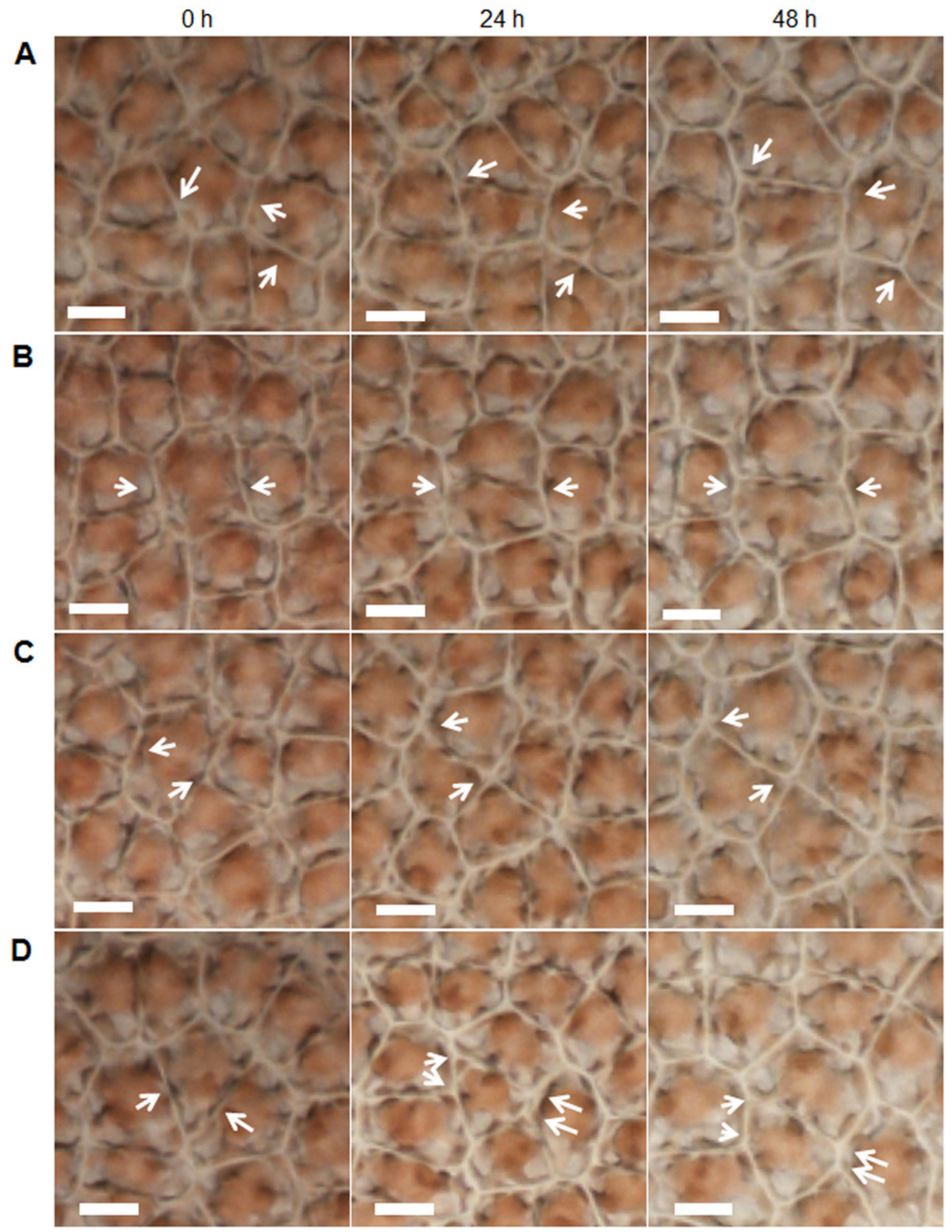

E

F

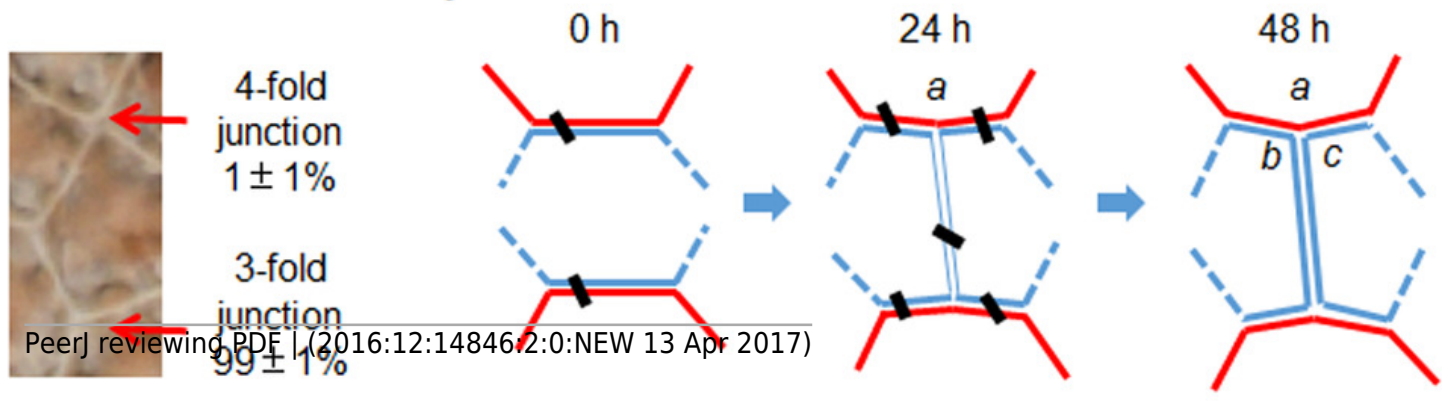

